\title{
A RANK THEOREM FOR INFINITE DIMENSIONAL SPACES
}

\author{
J. P. HOLMES
}

ABSTRACT. Suppose $X$ is a Banach space, $U$ is an open set of $X$ containing 0 , and $f$ is a continuously differentiable function from $U$ into $X$ satisfying $f(0)=0$ and $f^{\prime}(0)^{2}=f^{\prime}(0)$. An additional hypothesis is given for $f$ which, in case $X$ is finite dimensional, is equivalent to assuming $\operatorname{rank} f^{\prime}(x)=\operatorname{rank} f^{\prime}(0)$ for all $x$ in some neighborhood of 0 . Under this hypothesis one obtains a local factorization of $f$ into $h_{1} \circ$ $f^{\prime}(0) \circ h_{2}$ where each of $h_{1}$ and $h_{2}$ is a continuously differentiable homeomorphism. In addition there is a neighborhood of 0 in $f^{-1}(\{0\})$ which is the image of a continuously differentiable retraction. An application of these results to the theory of differentiable multiplications is given.

Suppose $X$ is a Banach space, $U$ is an open set of $X$ containing 0 , and $f$ is a continuously differentiable function from $U$ into $X$ satisfying $f(0)=0$ and $f^{\prime}(0)^{2}=f^{\prime}(0)$. An additional hypothesis is given for $f$ which, in case $X$ is finite dimensional, is equivalent to assuming rank $f^{\prime}(x)=\operatorname{rank} f^{\prime}(0)$ for all $x$ in some neighborhood of 0 . Under this hypothesis one obtains a local factorization of $f$ into $h_{1} \circ f^{\prime}(0) \circ h_{2}$ where each of $h_{1}$ and $h_{2}$ is a continuously differentiable homeomorphism. In addition there is a neighborhood of 0 in $f^{-1}(\{0\})$ which is the image of a continuously differentiable retraction. An application of these results to the theory of differentiable multiplications is given.

Condition I. There is a neighborhood $W$ of 0 in $X$ so that $\left(f^{\prime}(0) \mid f(W)\right)$ is one to one.

Condition II. There is a neighborhood $W$ of 0 in $X$ so that if $V$ is a neighborhood of 0 in $f^{\prime}(0)(X)$ then $f(V)$ is a neighborhood of 0 in $f(W)$.

Theorem 1. f satisfies Condition I if and only if $f$ satisfies Condition II.

Received by the editors May 6, 1974 .

AMS (MOS) subject classifications (1970). Primary 53B25; Secondary 22A 15. Key words and phrases. Rank theorem, differentiable semigroups. 
Proof. Let $N$ denote the image of $f^{\prime}(0) . N$ is closed since $f^{\prime}(0)^{2}=f^{\prime}(0)$ and hence is a Banach space. $\left(f^{\prime}(0) \circ f \mid N\right)^{\prime}(0)=\left(f^{\prime}(0) \circ f^{\prime}(0) \mid N\right)=\left(f^{\prime}(0) \mid N\right)$ is the identity function on $N$. Thus, by the inverse function theorem $[1, \mathrm{p}$. 268], there are neighborhoods $A$ and $B$ of 0 in $N$ so that $\left(f^{\prime}(0) \circ f \mid A\right)$ is a homeomorphism onto $B$ and $\left(f^{\prime}(0) \circ f \mid A\right)^{-1}$ is continuously differentiable on B.

Now suppose $f$ satisfies Condition $\mathrm{I}$ and $W^{\prime}$ is a neighborhood of 0 in $X$ so that $\left(f^{\prime}(0) \mid f\left(W^{\prime}\right)\right)$ is one to one. Choose a neighborhood $W$ of 0 in $X$ so that $W$ is contained in $W^{\prime}$ and $W \cap N$ is contained in $A$.

Suppose $V$ is a neighborhood of 0 in $N$ and $V$ is contained in $W$. $f^{\prime}(0)(f(V))$ is a neighborhood of 0 in $N$ since $V$ is contained in $A$. Let $C=$ $f^{\prime}(0)^{-1}\left(f^{\prime}(0)(f(V))\right) \cap f(W)$, and suppose $y$ is in $C . f^{\prime}(0)(y)$ is in $B$ so there is an $x$ in $A$ so that $f^{\prime}(0)(y)=f^{\prime}(0)(f(x))$. But each of $f(x)$ and $y$ is in $f(W)$ so $f(x)=y$ since $f(W)$ is contained in $f\left(W^{\prime}\right)$.

Suppose $f$ satisfies Condition II and choose $W^{\prime}$ so that if $V$ is contained in $W^{\prime}$ and $V$ is a neighborhood of 0 in $N$ then $f(V)$ is a neighborhood of 0 in $f\left(W^{\prime}\right)$. Since $f\left(A \cap W^{\prime}\right)$ is a neighborhood of 0 in $f\left(W^{\prime}\right)$ we may choose the neighborhood $W$ of 0 in $X$ so that $f(W)$ is contained in $f\left(A \cap W^{\prime}\right)$.

If each of $x$ and $y$ is in $f(W)$ there are members $z$ and $w$ of $A$ so that $f(z)=x$ and $f(w)=y$. If $f^{\prime}(0)(x)=f^{\prime}(0)(y)$ then $f^{\prime}(0)(f(z))=f^{\prime}(0)(f(w))$ and hence $z=w$ since each of $z$ and $w$ is in $A$. Thus $\left(f^{\prime}(0) \mid f(W)\right)$ is one to one.

Note. If $A$ is chosen as before and $C$ is a closed subset of $A$ then $(f \mid C)$ is a homeomorphism onto $f(C)$. This is a consequence of the following observations. If $\left\{a_{n}\right\}$ is a sequence in $C$ and $\left\{f\left(a_{n}\right)\right\}$ converges then $\left\{f^{\prime}(0) f\left(a_{n}\right)\right\}$ converges to some member $y$ of $f^{\prime}(0)(f(C))$. Thus $\left\{a_{n}\right\}$ converges to $\left(f^{\prime}(0) \circ f \mid A\right)^{-1}(y)$ and $(f \mid C)^{-1}$ is continuous.

Theorem 2. If $f(f(x))=f(x)$ for each $x$ in $U \cap f^{-1}(U)$ then $f$ satisfies Condition II.

This is a consequence of Lemma 3 in [2].

Theorem 3. If $f$ satisfies Condition $\mathrm{I}$ then there is a neighborhood $C$ of 0 in $X$ and continuously differentiable homeomorphisms $h_{1}$ and $h_{2}$, each from a neighborhood of 0 in $X$ onto a neighborhood of 0 in $X$, so that $(f \mid C)=$ $\left(h_{1} \circ f^{\prime}(0) \circ h_{2} \mid C\right)$.

Proof. Since $f$ satisfies Conditions I and II there is a neighborhood $W$ of 0 in $X$ so that if $V$ is a neighborhood of 0 in $N$ and $V$ is contained in $W$ then $f(V)$ is a neighborhood of 0 in $f(W)$ and $\left(f^{\prime}(0) \mid f(W)\right)$ is one to one. 
Choose neighborhoods $A$ and $B$ of 0 in $N$ so that $A$ is contained in $W$, $\left(f^{\prime}(0) \circ f \mid A\right)$ is a homeomorphism onto $B$, and $\left(f^{\prime}(0) \circ f \mid A\right)^{-1}$ is continuously differentiable on $B$.

Define $K_{2}$ on $W$ by $K_{2}(x)=f(x)+\left(I-f^{\prime}(0)\right)(x)$. (I denotes the identity function on $\left.X_{.}\right) \quad K_{2}^{\prime}(0)=I$ so by the inverse function theorem, there are neighborhoods $D$ and $E$ of 0 in $X$ so that $\left(K_{2} \mid D\right)=h_{2}$ is a homeomorphism onto $E$ and $f^{\prime}(0)(E)$ is contained in $B$.

Define $K_{1}$ by

$$
K_{1}(x)=\left(f^{\prime}(0) \mid f(A)\right)^{-1}\left(f^{\prime}(0)(x)\right)+\left(I-f^{\prime}(0)\right)(x)
$$

for each $x$ in $f^{\prime}(0)^{-1}(A \cap B) . K_{1}$ is well defined since $f(A)$ is contained in $f(W)$. If $x$ is in $A \cap B$ then

$$
(f \mid A) \circ\left(f^{\prime}(0) \circ f \mid A\right)^{-1}(x)=\left(f^{\prime}(0) \mid f(A)\right)^{-1}(x) .
$$

Thus $\left(f^{\prime}(0) \mid f(A)\right)^{-1} \circ f^{\prime}(0)$ is continuously differentiable on $\operatorname{dom}\left(K_{1}\right)$.

In particular, $K_{1}^{\prime}(0)=I$, so by the inverse function theorem there are neighborhoods $F$ and $G$ of 0 in $X$ so that $h_{1}=\left(K_{1} \mid F\right)$ is a homeomorphism onto $G$.

Choose $C$, a neighborhood of 0 in $X$, so that $C$ is contained in $D$, $f^{\prime}(0)\left(h_{2}(C)\right)$ is contained in $\operatorname{dom}\left(h_{1}\right)$, and $f(C)$ is contained in $f(A)$. (This last condition can be arranged since $f(A)$ is a neighborhood of 0 in $f(W)$.)

If $x$ is in $C$ then

$$
h_{1}\left(f^{\prime}(0)\left(b_{2}(x)\right)\right)=\left(f^{\prime}(0) \mid f(A)\right)^{-1}\left(f^{\prime}(0)(f(x))\right)=f(x) .
$$

Compare this conclusion with that of the rank theorem [1, p. 277].

Suppose $X$ is finite dimensional, $V$ is an open set of $X, x_{0}$ is in $V$, and $g$ is a continuously differentiable function from $V$ to $X$ satisfying $\operatorname{rank}\left(g^{\prime}(x)\right)=\operatorname{rank}\left(g^{\prime}\left(x_{0}\right)\right)$ for each $x$ in $V$. There is a linear homeomorphism $T$ from $X$ into $X$ so that the function $f$ defined by $f(x)=T\left(g\left(x^{2}+x_{0}\right)-g\left(x_{0}\right)\right)$ for each $x$ in $U=V-x_{0}$ satisfies $f^{\prime}(0)^{2}=f^{\prime}(0), f(0)=0$, and $\operatorname{rank}\left(f^{\prime}(x)\right)=$ $\operatorname{rank}\left(f^{\prime}(0)\right)$ for each $x$ in $U$. Thus, the following theorem may be applied to g.

Theorem 4. If $X$ is finite dimensional then $f$ satisfies Condition I if and only if $\operatorname{rank}\left(f^{\prime}(x)\right)=\operatorname{rank}\left(f^{\prime}(0)\right)$ for each $x$ in some neighborhood of 0.

Proof. Suppose $X$ is finite dimensional and $f$ satisfies Condition I. Choose homeomorphisms $h_{1}$ and $h_{2}$ as in the conclusion of Theorem 3 . Since $h_{1}^{\prime}(0)=h_{2}^{\prime}(0)=I$ there is a neighborhood $D$ of 0 in $X$ so that if $x$ is in $D$ then each of $h_{1}^{\prime}\left(f^{\prime}(0)\left(h_{2}(x)\right)\right)$ and $h_{2}^{\prime}(x)$ is invertible. If $x$ is in $D$ then $f^{\prime}(x)=h_{1}^{\prime}\left(f^{\prime}(0)\left(h_{2}(x)\right)\right) \circ f^{\prime}(0) \circ h_{2}^{\prime}(x)$, and hence $\operatorname{rank}\left(f^{\prime}(x)\right)=\operatorname{rank}\left(f^{\prime}(0)\right)$. 
Now suppose $X$ is finite dimensional, $D$ is a neighborhood of 0 in $X$, and $\operatorname{rank}\left(f^{\prime}(x)\right)=\operatorname{rank}\left(f^{\prime}(0)\right)$ for each $x$ in $D$. Let $N=f^{\prime}(0)(X)$ and $M=$ $\operatorname{ker}\left(f^{\prime}(0)\right)=\left(I-f^{\prime}(0)\right)(X)$. Define $h$ from $D$ into $N \times M$ by $h(x)=\left(f^{\prime}(0)(f(x))\right.$, $\left.\left(I-f^{\prime}(0)\right)(x)\right)$. Regard $N \times M$ as a Banach space in the usual way and note that $h^{\prime}(0)=f^{\prime}(0) \times\left(I-f^{\prime}(0)\right)$ is a linear homeomorphism onto $N \times M$. By the inverse function theorem, we may choose $E$ a neighborhood of 0 in $X$ so that $(h \mid E)$ is a homeomorphism onto a neighborhood $(0,0)$ in $N \times M,(b \mid E)^{-1}$ is continuously differentiable on $h(E)$, and $\left[(h \mid E)^{-1}\right]^{\prime}(x)=\left[h^{\prime}\left((h \mid E)^{-1}(x)\right)\right]^{-1}$ for each $x$ in $h(E)$.

If $x$ is in $E$ then $h^{\prime}(x)(n)=\left(f^{\prime}(0) \circ f^{\prime}(x)(n), 0\right)$ for each $n$ in $N . h^{\prime}(x)$ is invertible so $\operatorname{dim}\left(h^{\prime}(x)(N)\right)=\operatorname{dim}(N)=\operatorname{dim}(N \times\{0\})$. Thus $f^{\prime}(0) \circ f^{\prime}(x)(N)=$ $N$. This implies that each of $\left(f^{\prime}(0) \mid f^{\prime}(x)(X)\right)$ and $\left(f^{\prime}(x) \mid N\right)$ is one to one onto its image since $\operatorname{rank}\left(f^{\prime}(x)\right)=\operatorname{rank}\left(f^{\prime}(0)\right)$.

If $x$ is in $E$ then $f(x)=f\left(h^{-1}(h(x))\right)$. Hence, by the chain rule,

$$
\begin{aligned}
f^{\prime}(x) & =\left(f \circ b^{-1}\right)^{\prime}(b(x)) \circ b^{\prime}(x) \\
& =\left(D_{1} f \circ b^{-1}\right)(b(x)) \circ f^{\prime}(0) \circ f^{\prime}(x)+\left(D_{2} f \circ b^{-1}\right)(b(x)) \circ\left(I-f^{\prime}(0)\right) .
\end{aligned}
$$

So

$$
\begin{aligned}
\left(D_{2} f \circ b^{-1}\right)(b(x)) \circ & \left(I-f^{\prime}(0)\right)=f^{\prime}(x)-\left(D_{1} f \circ b^{-1}\right)(b(x)) \circ f^{\prime}(0) \circ f^{\prime}(x) \\
= & {\left[\left(f^{\prime}(0) \mid f^{\prime}(x)(X)\right)^{-1}-\left(D_{1} f \circ b^{-1}\right)(b(x))\right] f^{\prime}(0) \circ f^{\prime}(x) . }
\end{aligned}
$$

If $n$ is in $N$ then there is a $y$ in $N$ so that $f^{\prime}(0)(f(x)(y))=n$. Hence

$$
\begin{aligned}
& {\left[\left(f^{\prime}(0) \mid \operatorname{im}\left(f^{\prime}(x)\right)\right)^{-1}-\left(D_{1} f \circ b^{-1}\right)(h(x))\right](n)} \\
& \quad=\left(D_{2} f \circ b^{-1}\right)(b(x)) \circ\left(I-f^{\prime}(0)\right)(y)=0 .
\end{aligned}
$$

Thus $\left(D_{2} f \circ h^{-1}\right)(h(x)) \circ\left(I-f^{\prime}(0)\right)(y)=0$ for each $y$ in $X$ and $\left(D_{2} f \circ h^{-1}\right)(h(x))=0$ for each $x$ in $E$.

Choose neighborhoods $F$ and $G$ of 0 in $N$ and $M$, respectively, so that $G$ is convex and $F \times G$ is contained in $h(E)$. If each of $(x, y)$ and $(x, z)$ is in $F \times G$ then

$$
\begin{aligned}
f\left(b^{-1}(x, y)\right)-f\left(h^{-1}(x, z)\right)= & \int_{0}^{1} d t\left[\left(f^{\prime} \circ b^{-1}\right)^{\prime}(x, z+t(y-z))(0, y-z)\right] \\
= & \int_{0}^{1} d t\left[\left(D_{1} f \circ b^{-1}\right)(x, z+t(y-z))(0)\right. \\
& \left.\quad+\left(D_{2} f \circ b^{-1}\right)(x, z+t(y-z))(y-z)\right]=0
\end{aligned}
$$

since $(x, z+t(y-z))$ is in $h(E)$ for each $t$ in $[0,1]$. 
Choose a neighborhood $W$ of 0 in $X$ so that $h(W)$ is contained in $F \times G$. If each of $x$ and $y$ is in $W$ and $f^{\prime}(0)(f(x))=f^{\prime}(0)(f(y))$ then

$$
f(x)=f\left(b^{-1}(b(x))\right)=f\left(b^{-1}(l,(y))\right)=f(y)
$$

so $\left(f^{\prime}(0) \mid f(W)\right)$ is one to one.

The idea for the last part of this proof is contained in Dieudonne's proof of the rank theorem [1, p. 277].

Theorem 5. Suppose $f$ satisfies Condition I. There is a neighborbood $S$ of 0 in $X$ and a continuously differentiable function $q$ with domain $S$ so that $q(S)$ is a neighborbood of 0 in $f^{-1}(\{0\}), q \circ q=q$, and $(f \times q \mid S)$ is a homeomorphism onto a neighborhood of $(0,0)$ in $f(S) \times f^{-1}(\{0\})$.

Proof. By Conditions I and II we maychoose a neighborhood $W$ of 0 in $X$ so that $\left(f^{\prime}(0) \mid f(W)\right)$ is one to one and if $V$ is a neighborhood of 0 in $N$ contained in $W$ then $f(V)$ is a neighborhood of 0 in $f(W)$. Choose neighborhoods $A$ and $B$ of 0 in $N$ so that $\left(f^{\prime}(0) \circ f \mid A\right)$ is a homeomorphism onto $B,\left(f^{\prime}(0) \circ f \mid A\right)^{-1}$ is continuously differentiable on $B$, and $A$ is contained in $W$. Choose $C$ and $D$ neighborhoods of 0 in $X$ so that each of $C$ and $D$ is contained in $W$, $f(C)$ is contained in $f(A),\left(K_{1} \mid C\right)$ is a homeomorphism onto $D$, and $\left(K_{1} \mid C\right)^{-1}$ is continuously differentiable on $D$.

$f(A)$ is a neighborhood of 0 in $f(W)$ so $f(A) \times\left(I-f^{\prime}(0)\right)(W)$ is a neighborhood of $(0,0)$ in $f(W) \times\left(I-f^{\prime}(0)\right)(W)$. Suppose each of $(a, b)$ and $(c, d)$ is in $f(A) \times\left(I-f^{\prime}(0)\right)(W)$ and $a+b=c+d$. There are $x$ and $y$ in $A$ so that $f(x)=$ $a$ and $f(y)=c$. $d-b$ is in $\operatorname{im}\left(I-f^{\prime}(0)\right)=\operatorname{ker}\left(f^{\prime}(0)\right)$ so $f^{\prime}(0)(f(x))=f^{\prime}(0)(f(y))$. Thus $x=y, a=c$, and $b=d$ so $\left(+\mid f(A) \times\left(I-f^{\prime}(0)\right)(W)\right)$ is one to one.

Let $g$ be defined on $C$ by $g(x)=\left(f(x),\left(I-f^{\prime}(0)\right)(x)\right)$. Then $\left(K_{1} \mid C\right)=$ $(+\circ g \mid C)$. Hence, $(g \mid C)$ is one to one and $+(g(C))=D$.

$$
E=+^{-1}(!) \cap\left[f(A) \times\left(I-f^{\prime}(0)\right)(W)\right]
$$

is a neighborhood of $(0,0)$ in $f(W) \times\left(I-f^{\prime}(0)\right)(W)$. Suppose $e$ is in $E$. $+(e)=$ $K_{1}(z)$ for some $z$ in $C$ so $+(e)=+(g(z))$. But each of $e$ and $g(z)$ is in $f(A) \times$ $\left(I-f^{\prime}(0)\right)(W)$ so $g(z)=e$. Thus $g(C)$ contains $E$.

Choose $F$ and $G$ neighborhoods of 0 in $f(W)$ and $\left(I-f^{\prime}(0)\right)(W)$, respectively, so that $F \times G$ is contained in $g(C)$. Let $h=\left(K_{1}^{-1} \mid G\right)$, and define $q$ on $\left(I-f^{\prime}(0)\right)^{-1}(G)$ by

$$
q(x)=h\left(\left(I-f^{\prime}(0)\right)(x)\right)=g^{-1}\left(0, x-f^{\prime}(0)(x)\right) .
$$

If $x$ is in $\left(I-f^{\prime}(0)\right)^{-1}(G)$ then

$$
g(q(x))=g\left(g^{-1}\left(0, x-f^{\prime}(0)(x)\right)\right)=\left(0, x-f^{\prime}(0)(x)\right) .
$$


Thus $q(x)$ is in $f^{-1}(\{0\}) \cap\left(I-f^{\prime}(0)\right)^{-1}(G)$.

$$
q(q(x))=g^{-1}\left(0, q(x)-f^{\prime}(0)(q(x))\right)=g^{-1}(g(q(x)))=q(x)
$$

if $x$ is in $\left(I-f^{\prime}(0)\right)^{-1}(G)$ so $q \circ q=q$.

Suppose $x$ is in $g^{-1}(F \times G) \cap f^{-1}(\{0\})$.

$$
x=g^{-1}(g(x))=g^{-1}\left(0, x-f^{\prime}(0)(x)\right)=q\left(x-f^{\prime}(0)(x)\right) .
$$

Thus $\operatorname{im}(q)$ is a neighborhood of 0 in $f^{-1}(\{0\})$.

Since $h=\left(K_{1}^{-1} \mid G\right) h$ is continuously differentiable on $G, q$ is continuously differentiable on $\left(I-f^{\prime}(0)\right)^{-1}(G)$. Moreover, $q^{\prime}(0)=h^{\prime}(0) \circ\left(I-f^{\prime}(0)\right)$,

$$
h^{\prime}(0)=\left(\left[K_{1}^{\prime}\left(K_{1}^{-1}(0)\right)\right]^{-1} \mid \operatorname{im}\left(I-f^{\prime}(0)\right)\right)=\left(I-f^{\prime}(0) \mid \operatorname{im}\left(I-f^{\prime}(0)\right)\right)
$$

so $q^{\prime}(0)=I-f^{\prime}(0)$. From Lemma 3 of [2] there is a neighborhood $H$ of 0 in $\operatorname{im}\left(q^{\prime}(0)\right)$ so that $(q \mid H)$ is a homeomorphism onto a neighborhood of 0 in $\operatorname{im}(q)$.

Choose $S$ a neighborhood of 0 in $X$ so that $S$ is contained in $\left(I-f^{\prime}(0)\right)^{-1}(H \cap G)$ and $S \cap N$ is contained in $A$. Define $r$ on $f(S) \times$ $\left(I-f^{\prime}(0)\right)(S)$ by $r(x, y)=(x, q(y))$. By choice of $H, r$ is a homeomorphism onto a neighborhood of 0 in $f(S) \times q(S)$. $(f \times q \mid S)=(r \circ g \mid S)$. Thus $(f \times q \mid S)$ is a homeomorphism onto a neighborhood of $(0,0)$ in $f(S) \times q(S)$. This concludes the proof of Theorem 5 .

We will now indicate an application of the preceding theorems to the local theory of differentiable semigroups. Suppose $D$ is an open set of $X$ containing 0 , and $V$ is a continuously differentiable associative function from $D \times D$ into $X$ so that $V(0,0)=0$. Define $f$ on $D$ by $f(x)=V(x, 0)$. If each of $x$ and $f(x)$ is in $D$ then $f(f(x))=f(x)$. Thus, by Theorem $2, f$ satisfies Condition I. By Theorem 5 then there is a neighborhood $S$ of 0 in $X$ and a continuously differentiable function $q$ defined on $S$ so that $q \circ q=q$ and $\operatorname{im}(q)$ is a neighborhood of 0 in $f^{-1}(\{0\})$. By Lemma 3 of [2] there is a neighborhood $V$ of 0 in $Y=\operatorname{im}\left(q^{\prime}(0)\right)=\operatorname{im}\left(I-f^{\prime}(0)\right)$ so that $(q \mid V)$ is a homeomorphism onto a neighborhood of 0 in $\operatorname{im}(q),\left(q^{\prime}(0) \circ q \mid V\right)$ is a homeomorphism onto a neighborhood of 0 in $Y$, and $\left(q^{\prime}(0) \circ q \mid V\right)^{-1}$ is continuously differentiable. Define $W$ contained in $(Y \times Y) \times Y$ by

$$
W(x, y)=(q \mid V)^{-1}(V(q(x), q(y)))
$$

whenever $V(q(x), q(y))$ is in $q(V)$. The domain of $W$ is a neighborhood of $(0,0)$ in $Y \times Y, W$ is associative,

$$
\begin{aligned}
W(x, y) & =(q \mid V)^{-1} \circ\left(q^{\prime}(0) \mid q(V)\right)^{-1} \circ q^{\prime}(0) \circ V(q(x), q(y)) \\
& =\left(q^{\prime}(0) \circ q \mid V\right)^{-1} \circ q^{\prime}(0) \circ V(q(x), q(y))
\end{aligned}
$$

so $W$ is continuously differentiable on a neighborhood of $(0,0)$, and $q$ is a 
local isomorphism between the local differentiable semigroups $\left(f^{-1}(\{0\})\right.$, $\left.\left(V \mid f^{-1}(\{0\}) \times f^{-1}(\{0\})\right)\right)$ and $(Y, W)$.

In [3] it was shown that $\operatorname{im}(f)$ is locally the topological and algebraic product of a local Lie group and a left trivial semigroup. Horne in [4] began a study of differentiable semigroups with right zero. The above shows that $(X, V)$ is, near 0 , the topological product of the differentiable subsemigroups $f(D)$ and $f^{-1}(\{0\})$.

Can $V$ be reconstructed from its restriction to $(f(D) \times f(D)) \cup$ $\left(f^{-1}(\{0\}) \times f^{-1}(\{0\})\right) ?$

\section{REFERENCES}

1. J. Dieudonné, Foundations of modern analysis, Pure and Appl. Math., vol. 10, Academic Press, New York and London, 1960. MR 22 \#11074.

2. J. P. Holmes, Differentiable projections and differentiable semigroups, Proc. Amer. Math. Soc. 41 (1973), $251-254$.

3. - Differentiable semigroups, Colloq. Math. (to appear).

4. J. G. Horne, SL(2) has no $C^{1}$ extensions to a half space, Semigroup Forum 7 (1974), 286-291.

DEPARTMENT OF MATHEMATICS, AUBURN UNIVERSITY, AUBURN, ALABAMA 36830 